\title{
Temperature Dependence of Anuran Distortion Product Otoacoustic Emissions
}

\author{
Sebastiaan W. F. Meenderink ${ }^{1,2}$ and Pim van Dijk ${ }^{3,4}$ \\ ${ }^{1}$ Department of Otorhinolaryngology and Head E Neck Surgery, University Hospital Maastricht, \\ P.O. Box 5800, 6202 AZ Maastricht, The Netherlands \\ ${ }^{2}$ Institute for Brain and Behaviour, Maastricht University, Maastricht, The Netherlands \\ ${ }^{3}$ Department of Otorhinolaryngology, University Medical Center Groningen, P.O. Box 30.001, 9700 RB \\ Groningen, The Netherlands \\ ${ }^{4}$ School of Behavioral and Cognitive Neurosciences, University of Groningen, Groningen, The Netherlands
}

Received: 3 January 2006; Accepted: 15 April 2006; Online publication: 25 May 2006

\begin{abstract}
To study the possible involvement of energy-dependent mechanisms in the transduction of sound within the anuran ear, distortion product otoacoustic emissions (DPOAEs) were recorded in the northern leopard frog over a range of body temperatures. The effect of body temperature depended on the stimulus levels used and on the hearing organ under investigation. Low-level DPOAEs from the amphibian papilla (AP) were reversibly depressed for decreased body temperatures. Apparently, DPOAE generation in the AP depends on metabolic rate, indicating the involvement of active processes in the transduction of sound. In contrast, in the other hearing organ, the basilar papilla (BP), the effects of body temperature on DPOAEs were less pronounced, irrespective of the stimulus levels used. Apparently, metabolic rate is less influencing DPOAE generation. We interpret these results as evidence that no amplifier is involved in sound transduction in the BP. The passive functioning of the anuran BP would place this hearing organ in a unique position within tetrapod hearing, but may actually be beneficial to ectothermic species because it will provide the animal with a consistent spectral window, regardless of ambient or body temperature.
\end{abstract}

Correspondence to: Pim van Dijk · Department of Otorhinolaryngology $\cdot$ University Medical Center Groningen · P.O. Box 30.001, 9700 RB, Groningen, The Netherlands. Telephone: $+31-50-3612550$; fax: +31-50-3611698; e-mail: p.van.dijk@med.umcg.nl
Keywords: distortion product otoacoustic emissions, anuran, temperature dependence, passive hearing

\section{INTRODUCTION}

In frogs, the inner ear holds two anatomically separated papillae that are most sensitive to airborne sound: the amphibian papilla (AP) and the basilar papilla (BP). These papillae respond best to different frequency ranges and thus have complementary function. Prominent differences to the mammalian cochlea are the absence of differentiated groups of hair cells (i.e., no inner or outer hair cells) and the positioning of the hair cells on a rigid structure rather than over a flexible membrane. The AP is tonotopically organized, with the lowest $(0.1 \mathrm{kHz})$ and the highest $(1.2 \mathrm{kHz})$ frequencies being represented rostrally and caudally, respectively. Frequencies above $1.2 \mathrm{kHz}$ primarily excite the BP (e.g., 1.2$2.5 \mathrm{kHz}$ in Rana pipiens pipiens; Ronken 1991). This entire papilla seems to function as a single auditory filter (Ronken 1990; Van Dijk et al. 1997) with tuning probably arising from the mechanical properties of the papillar structures (i.e., no contribution of electrical tuning from the hair cells; Smotherman and Narins 1999). See Wever (1985) or Lewis and Narins (1999) for extensive descriptions of the anatomy and physiology of the two papillae. 
Although both papillae are capable of generating otoacoustic emissions (OAEs), there exist several differences between the two. The AP generates both spontaneous OAEs (Van Dijk et al. 1989) and distortion product OAEs (DPOAEs) (Van Dijk and Manley 2001). When using both low-level and intermediate-level stimuli, the resulting DPOAE amplitudes grow compressively with increasing stimulus levels (i.e., an $N \mathrm{~dB}$ change in both stimulus levels results in a change of the DPOAE amplitude $<N \mathrm{~dB}$; Meenderink and Van Dijk 2004), and they are highly vulnerable to anoxia (Van Dijk et al. 2003). These OAE characteristics are similar to those found in mammals, for which these are interpreted as evidence for the presence of a cochlear amplifier (Davis 1983). Such an amplifier is thought to boost the sound-induced vibrations of the inner ear structures, thus counteracting the dampening caused by the viscosity of the inner ear fluids (Gold 1948). The correspondence between these anuran and mammalian OAE characteristics is therefore suggestive for the presence of an amplifier in the frog AP (Meenderink and Van Dijk 2004).

In contrast to the AP, spontaneous OAEs seem absent in the BP (Van Dijk et al. 1989), although this papilla generates DPOAEs (Van Dijk and Manley 2001). These are, when evoked with low-level stimuli, only slowly affected by anoxic conditions (Van Dijk et al. 2003), Also, DPOAE input/output (I/O) curves from the BP only exhibit the aforementioned compressive growth over a narrow range of intermediate stimulus levels. For low-level stimuli, the slope of the I/O curve is clearly steeper (slope $>1 \mathrm{~dB} / \mathrm{dB}$ ), indicating expansive rather than compressive growth (Meenderink and Van Dijk 2004). These different OAE properties have been interpreted as evidence for the absence of an amplifier in this hearing organ; seemingly, the BP functions as a passive auditory receiver (Meenderink and Van Dijk 2004). If true, the passive nature of the $\mathrm{BP}$ places this hearing organ in a unique position within tetrapod hearing: no other tetrapod ear lacks an inner ear amplifier.

The aim of the present study was to test whether DPOAE generation in the AP and the BP involves an active amplifier, respectively. This was performed by recording DPOAEs from both papillae over a range of body temperatures. Because active processes are energy dependent, the amplifier is energy consuming. This energy must come from metabolism and hence will depend on temperature. Therefore, it is to be expected that the generation of DPOAEs is temperature dependent when an amplifier is involved in their generation. If the hypothesis is true, we expect to see a clear temperature dependence of low-level DPOAEs from the AP and less dependence for DPOAEs from the BP.

\section{MATERIALS}

Distortion product otoacoustic emissions (DPOAEs) were recorded from nine ears of five northern leopard frogs, $R$. pipiens pipiens [body weight 42.6$62.0 \mathrm{~g}$ (mean $52.5 \mathrm{~g}$ ) ]. To immobilize the frog, it was anesthetized with an intramuscular injection of a pentobarbital sodium solution (diluted Nembutal; effective dosage $60 \mathrm{mg} / \mathrm{kg}$ body weight) in one of the hind limbs. DPOAEs were monitored using a custombuilt probe that was carefully placed against the skin surrounding the tympanic membrane. A tight seal between the probe and the skin was obtained using Vaseline. To monitor the frog's temperature, a small, K-type thermocouple, connected to a thermocouple reader (Stanford Research Systems, SRS: SR630), was placed inside the frog's oral cavity. Recordings were made inside a sound-attenuating chamber, with the frog placed between wet gauze to prevent dehydration and to facilitate cutaneous respiration.

All procedures were approved by the animal experiment committee of the Maastricht University.

\section{DPOAE recording}

The same experimental setup as described by Meenderink and Van Dijk (2004) was used to record DPOAEs. Briefly, the recording probe contained two miniature speakers (Aero Company: E-A-RTONE 3A) for stimulus delivery and a 1-in. high-sensitivity condenser microphone (Brüel \& Kjær, B\&K: type 4179) for emission recording. Emissions were evoked by simultaneously presenting two pure tones of slightly different frequencies $\left(f_{1}\right.$ and $f_{2}$, respectively) to the ear. Each of these tones was generated from a separate D/A channel (Tucker-Davis Technologies, TDT: RP2), attenuated (TDT: PA5) to set the desired stimulus level ( $L_{1}$ and $L_{2}$, respectively), and played from one of the speakers contained within the probe.

The microphone signal was passed through both a low-noise amplifier (B\&K: type 2660; amplification = $20 \mathrm{~dB}$ ) and a measuring amplifier (B\&K: type 2610; amplification $\geq 0 \mathrm{~dB}$ ) before being recorded on a computer disc using an A/D channel (TDT: RP2). Stimulus tone generation and signal recording were controlled by software written in Matlab (The Mathworks) and RPvds (TDT). To monitor DPOAEs during the experiments, the spectrum of the amplified microphone signal was viewed on-line using a spectrum analyzer (SRS: SR760).

All DPOAE recordings were performed with $f_{2} / f_{1}=$ 1.1 , and stimulus frequency $f_{1}$ was chosen such that an integer number of sample points held exactly 100 periods of this tone. These periodic blocks of sample points also held an integer number of periods for the $f_{2}$ stimulus tone (110 periods) and for all DPOAEs 
(e.g., 90 periods for $2 f_{1}-f_{2}$ and 120 periods for $2 f_{2}-$ $f_{1}$ ). A series of these periodic blocks was recorded for each combination of stimulus tones. Artifact-free blocks (using a level-crossing artifact-rejection method) were averaged, and from this average, DPOAEs and noise were calculated using Fourier analysis.

In each ear investigated, the entire experiment consisted of the recording of several sets of DPOAE recordings, each set obtained at a different body temperature. A single set of DPOAE recordings entailed the mapping of the level dependence of DPOAEs in both the AP and the BP. Hereto, two $L_{1}$, $L_{2}$ maps were recorded, one from each papilla. Each $L_{1}, L_{2}$ map consisted of 81 recordings at different combinations of the two stimulus tones; $f_{1}$ and $f_{2}$ were fixed, whereas $L_{1}$ and $L_{2}$ were varied independently and randomly over a 32- $\mathrm{dB}$ range in 4- $\mathrm{dB}$ steps (Meenderink and Van Dijk 2005). For each ear, two stimulus frequency combinations were chosen to approximately match those combinations that resulted in maximum DPOAE amplitude in the AP and the BP, respectively. These frequencies were not varied during the entire experiment. For all ears, the two stimulus levels varied between 53 and $85 \mathrm{~dB}$ SPL in the AP and between 56 and $88 \mathrm{~dB}$ SPL in the BP.

\section{Experimental protocols}

Approximately $30 \mathrm{~min}$ after the administration of the anesthesia, the frog was transferred to the soundattenuating chamber. After placing the recording probe and the thermocouple, a single set of DPOAE recordings was obtained with the frog's body temperature at room temperature (approximately $21^{\circ} \mathrm{C}$ ). Subsequently, the animal was taken out of the setup and placed in a small plastic container. This container was then floated in a larger tank of cold water $\left(8^{\circ} \mathrm{C}\right)$ for $10 \mathrm{~min}$, which cooled the frog to a temperature of approximately $12^{\circ} \mathrm{C}$. After cooling, the frog was placed on a custom-built thermopad, and the recording probe and thermocouple were replaced. In potential, this moving of the frog may result in changes in DPOAE amplitudes because of slightly different placements of the recording probe. However, calibration data of the speakers show no large changes before and after moving the frog, suggesting a similar placement of the probe. In addition, repetitive DPOAE measurements in the same frog ear over the course of several days are very similar (Meenderink, personal observations). This indicates that moving the frog for cooling will not result in large effects on the recorded DPOAEs. The temperature of the thermopad could be controlled actively by flowing water of known temperature (using a LAUDA RE106 thermobath) through a maze of tubes integrated in the pad, thus controlling the frog's body temperature. Single sets of DPOAEs were recorded at different temperature settings (overall range $12-23^{\circ} \mathrm{C}$ ) after allowing the body temperature to stabilize for at least $5 \mathrm{~min}$ at each temperature setting.

To verify whether any of the observed effects were the result of the relatively rapid cooling of the frog, we also obtained sets of DPOAEs in one frog that was cooled more slowly $\left(\leq 0.3^{\circ} \mathrm{C} / \mathrm{min}\right)$. In addition, another animal was rapidly cooled, after which its body temperature was maintained at $12^{\circ} \mathrm{C}$ for 1 $\mathrm{h}$ before reheating was started. The results from these experiments were similar to all other results, indicating that the observed effects were not greatly affected by the rate of body temperature change.

\section{RESULTS}

The effects of body temperature on DPOAEs were similar in all ears studied. An example of these effects is illustrated in Figure 1. This figure shows both the amplitude (represented by equal-amplitude contour lines) and the phase (represented by arrows) for DPOAEs obtained in a single ear. At room temperature $\left(22^{\circ} \mathrm{C}\right.$; left column in Fig. 1$), L_{1}, L_{2}$ maps could be divided in two principal regions that showed a different stimulus level dependence of DPOAE amplitude. This difference is illustrated by the different shape of the equal-amplitude contour lines. When at least one of the two stimulus levels was relatively large, contour lines were approximately straight lines, with consecutive lines being parallel to each other. Note that the slope of these contour lines depended on the emission frequency (relative to the stimulus frequencies). For DPOAEs at $2 f_{1}-f_{2}$ (Fig. 1a, g), slopes approximate $L_{2} / L_{1}=-2 \mathrm{~dB} / \mathrm{dB}$, whereas for $2 f_{2}-f_{1}$, the slopes are close to $L_{2} / L_{1}=$ $-0.5 \mathrm{~dB} / \mathrm{dB}$ (Fig. 1d, j). In contrast, when both stimulus levels were relatively small, contour lines were convex and encompassed a low-level DPOAE ridge along which emission amplitude varied only marginally. This ridge covers a larger $L_{1}, L_{2}$ area in the AP compared to the BP (compare Fig. 1a and g), which is caused by the different slopes of low-level DPOAE I/O curves from the two papillae, respectively. I/O curves obtained from the AP show compressive decline $(<1 \mathrm{~dB} / \mathrm{dB})$ with decreasing stimulus levels, resulting in readily detectable emissions for relatively low stimulus intensities. In contrast, I/O curves recorded from the BP decline faster $(>1 \mathrm{~dB} /$ $\mathrm{dB}$ ) with decreasing stimulus levels. Only for a small interval of intermediate stimulus levels drops the decline below $1 \mathrm{~dB} / \mathrm{dB}$. As a consequence, BPDPOAEs are indiscernible from noise for low-level 

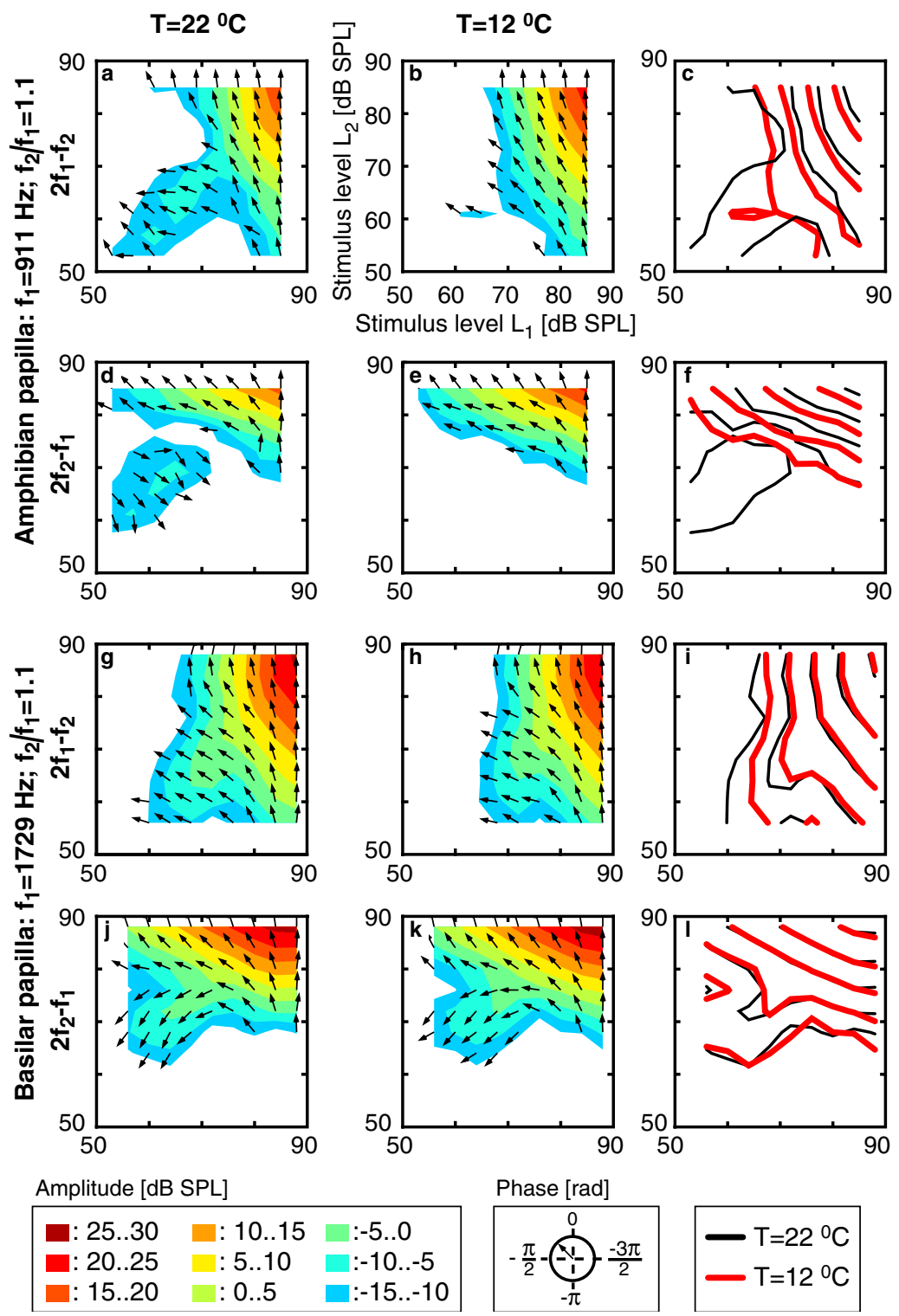

FIG. 1. Several input/output plots for DPOAE at $2 f_{1}-f_{2}$ and $2 f_{2}-f_{1}$ obtained in one frog. The different panels represent different stimulus frequencies and/or body temperatures (see labeling in the figure). $L_{1}$, $L_{2}$ maps show isoamplitude contour lines as function of the two stimulus levels. Contour lines are drawn at 5-dB intervals, with the appropriate levels given in the key. The arrows overlying the contour lines represent the phase of the DPOAEs, with one full counterclockwise rotation indicating a $2 \pi$ lag. In each $L_{1}, L_{2}$ map, phase is relative

stimuli that evoke AP-DPOAEs still exceeding the noise floor (Meenderink and Van Dijk 2004). For the phase data, $L_{1}, L_{2}$ maps could be divided into the same to stimulus level-dependent regions as for the DPOAE amplitude. Within each of these regions, DPOAE phase was relatively constant, whereas between them, the phase was different. We will refer to DPOAEs evoked with relatively small stimulus levels as low-level DPOAEs and to DPOAEs evoked with one or both stimulus levels relatively large as high-level DPOAEs. to the phase at the highest $L_{1}, L_{2}$ combination, which was arbitrarily set to zero. The left column shows data obtained with body temperature at $T=22^{\circ} \mathrm{C}$, whereas the center column shows results obtained when the recordings from the left column were repeated at a lower body temperature $\left(T=12^{\circ} \mathrm{C}\right)$. In the right column, data from the other two columns are redrawn (but with contour lines at 10-dB intervals) to facilitate graphical comparison between the DPOAE amplitudes recorded at the two body temperatures.

When body temperature was decreased to $12^{\circ} \mathrm{C}$ (Fig. 1, center column), the $L_{1}, L_{2}$ maps obtained from the AP (Fig. 1b, e) were markedly different from those obtained at room temperature. At this body temperature, the convex low-level DPOAEs were absent. In contrast, the high-level DPOAEs were still present and comparable in level to the emissions recorded at $22^{\circ} \mathrm{C}$ (Fig. 1c, f). For example, for the $L_{1}$, $L_{2}$ combinations given by $L_{1} \geq 70 \mathrm{~dB}$ SPL and $L_{2} \geq 65$ dB SPL in Figure 1a and b, the mean $2 f_{1}-f_{2}$ DPOAE amplitude increased by $3.01 \mathrm{~dB}$ (standard deviation, 
TABLE 1

\begin{tabular}{|c|c|c|c|c|}
\hline \multicolumn{5}{|c|}{ Temperature dependence of DPOAE levels at $2 f_{1}-f_{2}$ and $2 f_{2}-f_{1}$} \\
\hline & \multicolumn{2}{|c|}{ Amphibian papilla } & \multicolumn{2}{|c|}{ Basilar papilla $\left(\mathrm{dB} /{ }^{\circ} \mathrm{C}\right)$} \\
\hline & $\left(d B /{ }^{\circ} C\right)$ & $Q_{10}$ & $\left(d B /{ }^{\circ} C\right)$ & $Q_{10}$ \\
\hline High-level DPOAE & $-0.02( \pm 0.52)$ & 1.0 & $-0.01( \pm 0.50)$ & 1.0 \\
\hline Low-level DPOAE & $0.60( \pm 0.34)$ & 4.0 & $0.23( \pm 0.35)$ & 1.7 \\
\hline
\end{tabular}

The rate of amplitude change, averaged across subjects, is indicated in $\mathrm{dB} /{ }^{\circ} \mathrm{C}$, with the standard deviation given between parentheses. In addition, the thermal $Q_{10}$ values that correspond to the average rates are given. Each $Q_{10}$ equals the ratio of the intensities $I_{1}$ and $I_{2}$ measured at temperatures $10^{\circ} \mathrm{C}$ apart: $Q_{10}=10^{\text {(SPL2 }-}$ SPL1)/10, with $\mathrm{SPL}_{1}$ and $\mathrm{SPL}_{2}$ expressed in $\mathrm{dB}$.

$2.56 \mathrm{~dB}$ ) with the $10^{\circ} \mathrm{C}$ temperature decrease. For $2 f_{2}-$ $f_{1}$, this temperature decrease resulted in a mean amplitude increase of $3.16 \pm 1.91 \mathrm{~dB}$ (averaged for $L_{1}, L_{2}$ combinations given by $L_{1} \geq 56$ and $L_{2} \geq 84 \mathrm{~dB}$ SPL). Notice that the decreasing body temperature actually resulted in an increase in the high-level DPOAEs. In the $\mathrm{BP}$, the $10^{\circ} \mathrm{C}$ decrease in body temperature did not result in clear changes of DPOAE amplitude for any stimulus level combination represented in the $L_{1}, L_{2}$ maps (Fig. 1h, k). Here, both low-level and high-level DPOAEs were clearly detectable. Also, within both of the $L_{1}, L_{2}$ maps, the absolute DPOAE levels changed only marginally. For $2 f_{1}-f_{2}$, the average $\left(L_{1} \geq 68\right.$ and $L_{2} \geq 60$ dB SPL) DPOAE level decreased by $1.23 \pm 1.26 \mathrm{~dB}$ with the $10^{\circ} \mathrm{C}$ drop in body temperature (Fig. 1i), whereas for $2 f_{2}-f_{1}\left(L_{1} \geq 58\right.$ and $L_{2} \geq 68 \mathrm{~dB}$ SPL $)$, this was $0.39 \pm 1.22 \mathrm{~dB}$ (Fig. 11).

Table 1 displays the rate of DPOAE amplitude change as a function of body temperature averaged across subjects. A clear and consistent increase of DPOAE amplitude was only observed for low-level DPOAEs from the AP, with average thermal $Q_{10}=4.0$. Low-level DPOAEs from the BP also increased with increasing temperature, but the corresponding thermal $Q_{10}$ was only 1.7 on average. In both papillae, high-level DPOAE amplitudes did not show a consistent dependence on body temperature.

\section{DISCUSSION}

These results have important implications concerning the presence of an inner ear amplifier within the anuran ear. We use the term inner ear amplifier rather than cochlear amplifier because not all species whose ears support an amplification mechanism have cochleae. The inner ear amplifier is an active mechanism, which derives its energy from metabolism. Consequently, its functioning must depend on temperature. Moreover, the amplitude of DPOAEs that are generated or boosted by an active amplifier must also depend on body temperature. The relatively weak temperature dependence of DPOAE amplitude from the BP suggests that these emissions may not involve an active inner ear amplifier. In contrast, the temperature dependence of low-level DPOAEs from the AP is consistent with the presence of an active amplification mechanism in this hearing organ.

These conclusions are consistent with other observations on the difference between the $\mathrm{AP}$ and the $\mathrm{BP}$ : (1) the generation of spontaneous OAEs is limited to the AP only (Van Dijk and Manley 2001); (2) low-level DPOAEs from the AP show a higher vulnerability to physiological injuries than low-level DPOAEs from the BP (Van Dijk et al. 2003); and (3) characteristics of auditory nerve fibers that innervate the AP depend on body temperature, whereas fibers contacting the BP exhibit no such dependence (Stiebler and Narins 1990). These differences suggest an important difference in the functioning of the two hearing papilla.

An additional difference between the AP and the $\mathrm{BP}$ is the growth rate of DPOAE amplitude at very low stimulus amplitudes. In the BP, these emission amplitudes grow expansively (slope $>1 \mathrm{~dB} / \mathrm{dB}$ ) with increasing stimulus levels. In contrast, in the AP and in the hearing organs of most other tetrapods (reviewed in Meenderink and Van Dijk 2004), amplitudes of low-level DPOAEs grow compressively with a slope smaller than $1 \mathrm{~dB} / \mathrm{dB}$. Perhaps the compressive DPOAE growth is a universal property of the inner ear amplifier.

In addition to the temperature effects on the inner ear amplifier, other passive mechanisms may also be affected by the changed body temperature, thus changing the emission levels. For instance, decreasing the body temperature in rats reduces the compliance of the middle ear (Geal-Dor et al. 1997), resulting in a small conductive loss with an airborne gap of $6 \mathrm{~dB}$ (Khvoles et al. 1998). This conductive loss will reduce both the amplitudes of the two stimulus tones upon entering the inner ear, as well as the emission levels that are transmitted to the external ear. For low-level DPOAEs from the BP, we did find a small temperature dependence (Table 1). Such small changes may be explained by changes in passive sound-conductive properties of the inner ear 
structures. For example, assume that the viscosity of the inner ear fluids is the prominent cause for the inner ear's impedance. Then, for a constant driving force (i.e., constant stimulus level), DPOAE amplitude will decrease inversely proportional to the viscosity increase of the inner ear fluids. If the inner ear was filled with water, which has a thermal $Q_{10} \approx 1.3$, DPOAE intensity would thus have a thermal $Q_{10} \approx 1.3^{2} \approx$ 1.7.

Also, all the temperature-dependent changes that may affect the conduction of sound into and out of the ear (e.g., middle ear conductivity, viscosity of the inner ear fluids) will result in more or less similar DPOAE changes across different stimulus parameters. Because DPOAEs from the AP and the BP show different sensitivity to changes in body temperature, "overall" passive changes in the ear cannot account for the observed temperature effects. Rather, it seems that body temperature directly influences some mechanism that is exclusively at work in the AP. Given the magnitude of the changes with temperature, in combination with the similarity between mammalian DPOAEs and AP-DPOAEs, the most likely mechanism explaining the observed temperature dependence is the inner ear amplifier, whose functioning is most critical for low-level stimuli.

Several studies have explored the effects of body temperature on transient-evoked OAEs and/or DPOAEs in mammals (Ferber-Viart et al. 1995; Noyes et al. 1996; Veuillet et al. 1997; Khvoles et al. 1998). Results from these studies indicate that if changes in body temperature affect OAEs, this primarily occurs for emissions evoked with moderate-to-low stimulus intensities, consistent with our findings in the frog. In mammals, there seems to be a range of temperatures (around the normal body temperature) wherein OAEs are not correlated with temperature. Outside this temperature range, both for hypo- and hyperthermia, the amplitudes of low-level emissions are depressed. It is not clear from our results whether a similar range of body temperatures is present wherein low-level DPOAEs from the AP are uncorrelated with body temperature.

Even if the decreased temperature sensitivity of DPOAEs from the BP results from the absence of an inner ear amplifier, this does not imply that this auditory organ is functioning fully passively. An active $\mathrm{I} / \mathrm{O}$ system is defined by the fact that the output signal contains energy that does not derive directly from the input signal. In hair cells, the input is the deflection of the hair bundles, and the output is a transduction current through the ion channels in the hair cell's membrane. This current is gated by the input signal, but its energy is derived from an ion concentration gradient across the cell's membrane. Consequently, the forward mechanoelectrical trans- duction in hair cells is an active mechanism. Similar to forward transduction, reverse (electromechanical) transduction in hair cells is also active. The moderate sensitivity of DPOAEs from the BP to temperature changes implies that apparently both forward and reverse transductions are not directly involved in their generation. Rather, DPOAEs in the BP may result from nonlinear reflectances, which could involve the nonlinear stiffness of hair bundles (Howard and Hudspeth 1988).

As an alternative to a passive hearing organ, it may be that the energy supply sustaining an active mechanism in the BP is not dependent on body temperature. The presence of such an energy store could explain both the delayed vulnerability (Van Dijk et al. 2003) and temperature sensitivity of BPDPOAEs. In effect, a reserve energy supply would sustain an active mechanism for a short period of time after metabolism ceases. However, this does not offer an explanation for the absence of spontaneous OAEs or the aberrant growth rate of DPOAE I/O curves in the BP. Also, a much slower rate of cooling, or even maintaining body temperature at $12^{\circ} \mathrm{C}$ for a prolonged period, did not result in a more pronounced temperature dependence of BP-DPOAEs, whereas such experimental paradigms should have depleted the energy reserve. Finally, to the best of our knowledge, BP hair cells are not different from AP hair cells in that neither contains special structures or large numbers of mitochondria that could function as the pool for such an energy supply.

Our results place the $\mathrm{BP}$ of anurans in a unique position among tetrapods; distinct from the AP and from the inner ears of all other land-living vertebrates, the BP may be the only hearing organ without active feedback.

The question arises on how the frog may benefit from a passive ear. Seemingly, the absence of active amplification would reduce both the sensitivity and the frequency selectivity of the papilla. However, in a statistical analysis of nerve fiber tuning properties, Ronken (1991) showed that there is no clear difference between the sensitivity and the frequency selectivity of nerve fibers innervating the AP and the BP. Thus, assuming that these measures accurately reflect the hearing sensitivity for normal physiological conditions, there is no obvious difference between the active $\mathrm{AP}$ and the passive $\mathrm{BP}$ in this respect. Apparently, the BP derives its sensitivity and specificity via a mechanism other than amplification. Because the entire BP seems to function as a single auditory filter, the conjoint functioning of all its hair cells may achieve this. The absence of a temperature-dependent amplification mechanism within the frog's BP may even be beneficial. Given the ectothermic nature of amphibians, their body temperatures match the 
ambient temperature, which may fluctuate considerably. The absence of a temperature-dependent inner ear amplifier makes the functioning of the BP not hindered by these temperature fluctuations: the spectral window of auditory input is consistently transduced in the BP, independent of ambient and/ or body temperature.

\section{ACKNOWLEDGMENTS}

This work was supported by grants from the Netherlands Organization for Scientific Research (NWO) and the Heinsius Houbolt Foundation.

\section{REFERENCES}

DAvIs H. An active process in cochlear mechanics. Hear. Res. 9:7990, 1983.

Ferber-Viart C, Savourey G, Garcia C, Duclaux R, Bittel J, Collet L. Influence of hyperthermia on cochlear micromechanical properties in humans. Hear. Res. 91:202-207, 1995.

Geal-Dor M, Khvoles R, Sohmer H. Cooling induces a decrease in middle ear compliance. J. Basic Clin. Physiol. Pharmacol. 8:127-132, 1997.

Gold T HEaring II. the physical basis of the action of the cochlea. Proc. R. Soc. Edinb. B135:492-498, 1948.

Howard J, HudsPeth AJ. Compliance of the hair bundle associated with gating of mechanoelectrical transduction channels in the bullfrog's saccular hair cell. Neuron. 1:189-199, 1988.

Khvoles R, Freeman S, Sohmer H. Effect of temperature on the transient evoked and distortion product otoacoustic emissions in rats. Audiol. Neuro-otol. 3:349-360, 1998.

LEWIS ER, NARINS PM. The acoustic periphery of amphibians. In:
Fay RR and Popper AN (eds) Comparative Hearing: Fish and Amphibians. New York, Springer-Verlag, pp. 101-154, (1999).

MeEnderink SWF, VAN DiJK P. Level dependence of distortion product otoacoustic emissions in the leopard frog, Rana pipiens pipiens. Hear. Res. 192:107-118, 2004.

Meenderink SWF, VAn Dijk P. Characteristics of distortion product otoacoustic emissions in the frog from $L_{1}, L_{2}$-maps. J. Acoust. Soc. Am. 118:279-286, 2005.

Noyes WS, McCaffrey TV, Fabry DA, Robinette MS, Suman VJ. Effect of temperature elevation on rabbit cochlear function as measured by distortion-product otoacoustic emissions. Otolaryngol. Head Neck Surg. 115:548-552, 1996.

Ronken DA. Basic properties of auditory-nerve responses from a 'simple' ear: The basilar papilla of the frog. Hear. Res. 47:6382, 1990.

Ronken DA. Spike discharge properties that are related to the characteristic frequency of single units in the frog auditory nerve. J. Acoust. Soc. Am. 90:2428-2440, 1991.

SMOtherman MS, Narins PM. Potassium currents in auditory hair cells of the frog basilar papilla. Hear. Res. 132:117-130, 1999.

Stiebler IB, Narins PM. Temperature-dependence of auditory nerve responses properties in the frog. Hear. Res. 46:63-82, 1990.

VAN DIJK P, Manley GA. Distortion product otoacoustic emissions in the tree frog Hyla cinerea. Hear. Res. 153:14-22, 2001.

VAn Dijk P, Wit HP, Segenhout JM. Spontaneous otoacoustic emissions in the European edible frog (Rana esculenta): Spectral details and temperature dependence. Hear. Res. 42:273-282, 1989.

Van Dijk P, Wit HP, Segenhout JM. Dissecting the frog inner ear with Gaussian noise. I. Application of high-order Wiener-kernel analysis. Hear. Res. 114:229-242, 1997.

Van Dijk P, Narins PM, Mason MJ. Physiological vulnerability of distortion product otoacoustic emissions from the amphibian ear. J. Acoust. Soc. Am. 114:2044-2048, 2003.

Veuillet E, Gartner M, Champsaur G, Neidecker J, Collet L. Effects of hypothermia on cochlear micromechanical properties in humans. J. Neurol. Sci. 145:69-76, 1997.

WeVer EG. The Amphibian Ear. New Jersey, Princeton University Press, (1985). 